\title{
Translation, Adaptation and Cross Language Validation of Tinnitus Handicap Inventory in Urdu
}

\author{
Muhammad Aqeel and Ammar Ahmed \\ Department of Psychology, Foundation University Islamabad, Rawalpindi Campus, Rawalpindi, Pakistan
}

Received May 16, 2017

Revised July 31,2017

Accepted September 29, 2017

Address for correspondence

Muhammad Aqeel, Mr

Department of Psychology,

Foundation University Islamabad,

Rawalpindi Campus, New Lalazar,

Rawalpindi 46000, Pakistan

Tel +9203346278961

Fax +92-51-5151433

E-mail aqeel.1924@gmail.com
Background and Objectives: Tinnitus is characterized as a perception of numerous auditory sounds in absence of external stimulus. Tinnitus can have a considerable consequence on a person's quality of life, and is considered to be very complicated to quantify. The aim of this study was to investigate the reliability and validity of Urdu translation of the Tinnitus Handicap Inventory (THI) in Pakistan. It was designed to assess the presence of various auditory sounds without the external stimulus. Scale consisted of 25 items having three subscales functional, emotional, and catastrophic. Subjects and Methods: The study comprised into two stages, preliminary and main studies. The results of preliminary study revealed that the overall scale had high internal consistency [alpha coefficient of Urdu version of THI (THI$\mathrm{U})=0.99$, alpha coefficient of English version of $\mathrm{THI}=0.98]$. The overall scale had test-retest correlation over a fifteen days period of interval (0.99). Main study was performed on 110 tinnitus patients. The results of main study showed that the internal consistency and reliability of Urdu version was $(\alpha=0.93)$. The THI-U and its subscales demonstrated good internal consistency reliability ( $\alpha=0.81$ to 0.86 ). Results: High to moderate correlations were noted between tinnitus symptom ratings. A confirmatory factor analysis was used to validate the three subscales of $\mathrm{THI}-\mathrm{U}$, and high inter-correlations were found between the subscales also results revealed that a three-factor model for the THI-U was most tenable. The results displayed that the confirmatory factor analysis confirmed to validate the three subscales of THI-U. Conclusion: THI-U might present important information about precise facets of tinnitus distress along with diagnostic interviews in clinical practice.

J Audiol Otol 2018;22(1):13-19

KEY WORDS: Tinnitus Handicap Inventory · Tinnitus patients · Reproducibility of results . Translation · Confirmatory factor analysis.

\section{Introduction}

The word tinnitus is derived from Latin word tinnire meaning to ring [1]. The noises that can be heard which include hissing, thumping, whistling, buzzing ringing and so forth. It has been reported that around a quarter of adults experience tinnitus at any one time in their life [2]. The noises heard or perceived have alternating intensities along with differences in pitch, duration and loudness [3]. It has been mentioned that "tinnitus is not a kind of hallucination of psychiatric illness"

This is an Open Access article distributed under the terms of the Creative Commons Attribution Non-Commercial License (http://creativecommons.org/licenses/by-nc/4.0/) which permits unrestricted non-commercial use, distribution, and reproduction in any medium, provided the original work is properly cited.
[4]. Most of the reported occurrences of tinnitus may be associated to its multiple etiologies, like development of ear wax, infections in the middle ear, otosclerosis or cochlear hair cell damage, sometimes it has been reported that tinnitus can also be of idiopathic nature [5].

The self-rating questionnaires are used most of the time to study the impact of tinnitus. It helps in planning the treatment and addressing concerns of patients during the process of counseling. Self-rating questionnaires are also useful in evaluating and comparing treatment results. Also these questionnaires help in finding out symptoms and tinnitus-related history, and it enables the individual to describe the subjective impact of tinnitus. One of the most widely used and accepted questionnaires used in this area is the Tinnitus Handicap In- 
ventory (THI) [6].

THI is a widely employed to evaluate for tinnitus patients in all over the world. It has been translated and adapted in various languages globally for establish psychometric properties, some of which are Danish [7], Spanish [8], German [9], Brazilian Portuguese [10], Turkish [11], Italian [12], Chinese (Cantonese) [13], French [14], Kannada [15], Persian [16], and Hebrew [17].

Responses to the self-rating questionnaires are the most precise when administered in the native or local language of the individual. However, there has been no self-rating questionnaire in Urdu to assess handicap levels due to tinnitus. Urdu, the national language of Pakistan, is spoken by 60 to 80 million speakers approximately and is ranked as a fifth most spoken language comprising of 4.7 percent of the total world population, spoken mostly in the regions of South Asia, Pakistan and India [18].

Up till now, tinnitus-specific questionnaire has not been available in the Urdu language. The primary purpose of the present study was to investigate whether the Urdu translation of the Urdu version of THI (THI-U) is a reliable and valid measure for tinnitus-related problems in Pakistani tinnitus patients. Additionally, the study can serve as a general cross-cultural validation of the construct in question.

\section{Research design}

The present design was cross-sectional and employing a quantitative approach. This study was accomplished in 2 stages, i.e., preliminary and main studies.

\section{Subjects and Methods}

\section{Experimental process}

Current study was performed in two stages. Stage I dealt with the translation of THI-U, while stage II included the cross language validation and established psychometrics of THI scales. Standard back translation method was employed for translation and adaption aim from English language to Urdu language $[19,20]$. The translation process was accomplished in four steps 1 (translation); 2 (committee approach); 3 (back translation); and 4 (committee approach). The study protocol was approved by the Institutional Review Board of Medical and Psychology Center of Foundation University (FURC/IRB/38-A/2016). Written informed consent was obtained from all participants.

\section{Stage-I: standard back translation method}

\section{Part I: translation process}

Five bilingual professionals were approached for the Urdu translation process of THI. Professionals were instructed regarding the present research endeavor. Three Ear, Nose, and Throat (ENT) specialists, one psychologist and an audiologist were approached from different hospitals of Islamabad. Experts were instructed to focus on conceptual rather than factual translation and to use parsimonious language [1].

\section{Part 2: committee approach}

Committee of the professionals consisted on same five experts including ENT specialists $(n=3)$, clinical psychologists $(n=1)$, audiologist $(n=1)$, who were supervisors as well as researchers for the study. Experts selected the good and suitable items which were considered appropriate within the culture. After that the scale was provide to five other bilingual professionals for translating from Urdu language into English language.

\section{Part 3: back translation}

Five new professionals which were only experts in English language $(n=5)$ were approached to translate Urdu scale into English scale to ensure the accuracy and efficacy of Urdu translated scales. These bilingual experts were not part of the previous committee and therefore were not acquainted with the original wording of the English scale.

\section{Part 4: committee approach}

Second committee also comprised on five other bilingual subject professionals including supervisor of the study, clinical psychologist $(n=2)$, lecturer in English $(n=1)$, audiologist $(n=1)$ and the researcher himself. They were instructed to critically inspect back-translated items of English, and choose best items for the final scale.

\section{Stage-II: determination of psychometric properties of THI-U}

Statistical Package for Social Sciences (SPSS version 20, IBM Corp., Armonk, NY, USA) was employed to check cronbach alpha reliability, test-retest reliability, and item-total correlations of both English and Urdu scales.

\section{Part 1: instruments}

THI-U was devised by Newman, et al. [6], and used for translation. It is designed to measure tinnitus severity. It contains 25 items scored on a summated rating scale ranging from $\mathrm{No}=0$ to Always $=4$. On subscales number 1 (functional); 2 
(emotional); and 3 (catastrophic) scores range from 0-60. On scales numbers 1 (functional) consists on 12 items (12, 14, $18,15,4,1,24,20,9,13,2$, and 7) and the score range is $0-48$, which is measured dysfunction's mental and physical area. Scales numbers 2 (emotional) has 8 items $(6,16,10,22$, $21,3,25$, and 17) the score range is $0-32$. It is assessed affective responses to tinnitus, including anger, frustration, irritability, and depression. Scale number 3 (catastrophic) comprises on 5 items $(11,5,23,8$, and 19) with score range from $0-20$, which is investigated the most severe reaction to tinnitus such as desperation, loss of hope, inability to cope and fear of a grave disease. Higher scores on each subscales reveals more prevalence of tinnitus's severity. Low scores on each subscales shows low occurrence of tinnitus's severity. THI-U has good internal consistency for all sub scales $\alpha$ values range from $0.84-0.93$ [6]. Test re-test reliability of total score and subscales ranges from 0.84 to 0.94 [21].

\section{Part 2: sample}

For the determination of cross language validation the sam-

Table 1. Pearson product-moment correlation coefficient, mean and SD, range values associated with the test-retest administration of the THI-E and THI-U $(n=30)$

\begin{tabular}{|c|c|c|c|c|}
\hline THI-U & $\begin{array}{c}\text { Test } \\
\text { (English) }\end{array}$ & $\begin{array}{l}\text { Retest } \\
\text { (Urdu) }\end{array}$ & $\begin{array}{l}\text { Correlation } \\
\text { coefficients }\end{array}$ & p-value \\
\hline \multicolumn{3}{|c|}{ Functional (maximum score: 48) } & 0.99 & 0.000 \\
\hline Mean & 8.66 & 8.8 & & \\
\hline SD & 16.98 & 16.57 & & \\
\hline Range & $0-48$ & $0-48$ & & \\
\hline \multicolumn{3}{|c|}{ Emotional (maximum score: 32) } & 0.99 & 0.000 \\
\hline Mean & 4.4 & 4.93 & & \\
\hline SD & 9.74 & 11.08 & & \\
\hline Range & $0-28$ & $0-32$ & & \\
\hline \multicolumn{3}{|c|}{ Catastrophic (maximum score: 20 ) } & 0.97 & 0.000 \\
\hline Mean & 2.53 & 2.53 & & \\
\hline SD & 5.20 & 6.34 & & \\
\hline Range & $0-16$ & $0-20$ & & \\
\hline \multicolumn{3}{|c|}{ Total (maximum score: 100) } & 0.99 & 0.000 \\
\hline Mean & 15.60 & 16.26 & & \\
\hline SD & 31.78 & 33.79 & & \\
\hline Range & $0-92$ & $0-100$ & & \\
\hline
\end{tabular}

SD: standard deviation, THI-E: English version of Tinnitus Handicap Inventory, THI-U: Urdu version of Tinnitus Handicap Inventory ple of 30 students was randomly drawn from identical classes. Here we followed the translation procedure as given by students had the appropriate knowledge for both languages i.e., of 9th-10th grade English and Urdu. These students were selected from Fauji Foundation school of Islamabad. The core purpose to pick this sample was to confirm the cross language reliability and validation of THI scale. Tinnitus patients were not selected in pilot study because they have no knowledge of the English language particularly lower class patients of Pakistan then professionals choose that most suitable sample for pilot is 9th and 10th class students. For cross language validation sample $(\mathrm{n}=30)$ was selected. Thirty students were divided into two groups. In the first tryout two groups comprising of 30 school students 15 in each group were given the original inventory of the THI with translated version Urdu and English. Their responses were noted on translated scales of Urdu and English. In the second tryout after the 15 days interval the same 30 students were included to make their responses again, however in the revised try out second group of 30 students was given Urdu scale and English scale of the THI with the same instructions. This whole procedure was followed in order to identify any equivalence or discrepancy issues between the Urdu and English version of THI.

\section{Results}

External reliability for THI-U was examined by its test and retest reliability. Pearson correlation of the first and second test scores was based on three subscales (functional, emotional and catastrophic) and between the total scores of test and retest presented in Table 1. The Pearson correlation ranged between 0.80 and 0.99 . The Pearson correlation was greater than 0.97 for all subscales. The Pearson correlation for total score was 0.99 . The higher values of Pearson correlation indicate good test and retest reliability.

The internal consistency (Cronbach's $\alpha$ coefficient) of each subscale were reported (alpha coefficient of tinnitus handicap urdu subscales 1) functional $=0.99,2$ ) emotional $=0.99$, and 3) catastrophic $=0.96$ and Alpha coefficient of Tinnitus Handicap English subscales 1) functional $=0.98,2$ ) emotional $=0.97$, and 3) catastrophic $=0.92$ ), respectively. Each subscales had test-retest correlation of tinnitus handicap Urdu and English

Table 2. Reliability coefficients (Cronbach's $\alpha$ coefficient) of the THI-U and THI-E

\begin{tabular}{lcccc}
\hline & THI-U (a) & THI-E (a) & THI-U (item -total) & THI-E (item-total) \\
\hline THI-total (25 items) & 0.99 & 0.98 & $0.78-0.98$ & $0.57-0.98$ \\
Functional (12 items) & 0.99 & 0.98 & $0.79-0.95$ & $0.88-0.95$ \\
Emotional (8 items) & 0.99 & 0.97 & $0.73-0.99$ & $0.45-0.97$ \\
Catastrophic (5 items) & 0.96 & 0.92 & $0.67-0.98$ & $0.61-0.96$ \\
\hline
\end{tabular}

THI-E: English version of Tinnitus Handicap Inventory, THI-U: Urdu version of Tinnitus Handicap Inventory 
subscales 1) functional $=0.99,2$ ) emotional $=0.99$, and 3 ) catastrophic $=0.97$ (Table 2). As nearly all the items were showing high total item correlations and reliability, which indicated that it is a valid scale for research.

Main study included 110 tinnitus patients (males, $\mathrm{n}=70$; females, $n=40$ ), age ranged from 18 to 91 years, with the chief complaint of tinnitus due to conditions like stress, conductive hearing loss, vestibular Schwannoma, hypertension etc. between January 2016 and August 2016 from different private and public hospitals at the ENT and Audiology Department Hearts International Hospital and Alam Audiology Clinic, Rawalpindi and Lahore, Pakistan. Inclusion criteria for the subjects were based on experience of tinnitus for $\geq 3$ months, as well as tinnitus was constantly present during this duration either unilateral or bilateral. For this subjects were screened for tinnitus symptoms and hearing impairments. This study was performed in collaboration with the Psychology Department of Foundation University and Otolaryngologists/Audiologists Department Hearts International Hospital from Islamabad, Pakistan. All participants were native Urdu speakers and able to read and write Urdu. The study has been endorsed by the ethical committee of the Foundation University, Rawalpindi Campus of Institutional/ethical/ Review Board. The final THI-U scale has been administered to 110 tinnitus patients who completed the eligibility criteria with self perceived handicap due to tinnitus.

\section{Construct validity of THI-U}

The construct validity was established, by the total score on each subscale (functional, emotional, and catastrophic) was correlated with all the other subscales and also with the total of THI-U score. Results of correlation were obtained by the Pearson product moment method, the results of THI-U were significantly correlated with its subscales functional $(\mathrm{r}=0.96$, $p<0.001)$ emotional $(\mathrm{r}=0.92, p<0.001)$ and catastrophic $(\mathrm{r}=0.87, p<0.001)$. Also correlations were found to be significant $(p=0.000)$ across all three subscales and with the total THI-U score as shown in Table 3.

\section{Internal consistency reliability of THI-U}

The internal consistency reliability was calculated using the Cronbach's alpha for both the THI-U total and the three sub-

Table 3. Correlation matrix across the subscales and total for THI$U(n=110)$

\begin{tabular}{lcccc}
\multicolumn{1}{c}{ Scales } & 1 & 2 & 3 & 4 \\
\hline 1. THI-U total & - & $0.956^{* *}$ & $0.922^{* *}$ & $0.873^{* *}$ \\
2. Functional & & - & $0.816^{* *}$ & $0.755^{* *}$ \\
3. Emotional & & & - & $0.741^{* *}$ \\
4. Catastrophic & & & & - \\
\hline$* 0<0.01$. THI-U: Urdu version of Tinnitus Handicap Inventory
\end{tabular}

scales (i.e., functional, emotional, and catastrophic). Cronbach's alpha was high for the total THI-U (0.93), functional (0.86), emotional (0.80), and catastrophic subscale (0.81). Therefore, THI-U version was found to be reliable.

Item-total correlations for the total scale along with the three subscales are summarized in Table 4. The subscales of the THI were significantly correlated. It is apparent from the results that nearly all the items for THI-U have positive correlation with the total score demonstrating a highly significant internal consistency of the scale, only item No. 2 is having negative correlation with the overall scale.

\section{Confirmatory factor analysis}

A confirmatory factor analysis was performed to test whether the data could confirm the underlying variables represented by the subscales functional, emotional, and catastrophic of the translated version of the THI.

Table 5 displayed that the fit indices of the THI data were not perfect for the original factor structures (i,e., three-factor). Base on the modification indices for the three-factor model,

Table 4. Item-total correlations for the total scale along with its three subscales

\begin{tabular}{|c|c|c|c|c|}
\hline & $\begin{array}{c}\text { THI-U } \\
r\end{array}$ & $\begin{array}{c}\text { Emotional focus } \\
r\end{array}$ & $\begin{array}{c}\text { Functional } \\
r\end{array}$ & $\begin{array}{c}\text { Catastrophic } \\
r\end{array}$ \\
\hline THI-U1 & $0.592^{* *}$ & & $0.546^{* *}$ & \\
\hline THI-U2 & -0.031 & & 0.096 & \\
\hline THI-U3 & $0.570^{* *}$ & $0.645^{* *}$ & & \\
\hline THI-U4 & $0.628^{* *}$ & & $0.687^{* *}$ & \\
\hline THI-U5 & $0.789 * *$ & & & $0.738^{* *}$ \\
\hline THI-U6 & $0.586^{* *}$ & $0.648^{* *}$ & & \\
\hline THI-U7 & $0.635^{* *}$ & & $0.650^{* *}$ & \\
\hline THI-U8 & $0.639 * *$ & & & $0.766^{* *}$ \\
\hline THI-U9 & $0.750^{* *}$ & & $0.745^{* *}$ & \\
\hline THI-U10 & $0.730^{* *}$ & $0.777^{* *}$ & & \\
\hline THI-U1 1 & $0.717^{* *}$ & & & $0.807^{* *}$ \\
\hline THI-U12 & $0.730^{* *}$ & & $0.692^{* *}$ & \\
\hline THI-U13 & $0.550^{* *}$ & & $0.629 * *$ & \\
\hline THI-U14 & $0.717^{* *}$ & & $0.681^{* *}$ & \\
\hline THI-U15 & $0.713^{* *}$ & & $0.716^{* *}$ & \\
\hline THI-U16 & $0.542^{* *}$ & $0.630^{* *}$ & & \\
\hline THI-U17 & $0.326^{* *}$ & $0.402^{* *}$ & & \\
\hline THI-U18 & $0.717^{* *}$ & & $0.776^{* *}$ & \\
\hline THI-U19 & $0.538^{* *}$ & & & $0.688^{* *}$ \\
\hline THI-U2O & $0.705^{* *}$ & & $0.731^{* *}$ & \\
\hline THI-U21 & $0.629 * *$ & $0.699 * *$ & & \\
\hline THI-U22 & $0.736^{* *}$ & $0.734^{* *}$ & & \\
\hline THI-U23 & $0.619 * *$ & & & $0.787^{* *}$ \\
\hline THI-U24 & $0.556^{* *}$ & & $0.607^{* *}$ & \\
\hline THI-U25 & $0.680^{* *}$ & $0.668^{* *}$ & & \\
\hline
\end{tabular}


Table 5. Confirmatory factor fit indices

\begin{tabular}{ccccccccccc}
\hline Scale and factor & $\chi^{2}$ & $\mathrm{df}$ & $\chi^{2} / \mathrm{df}$ & $\mathrm{CFI}$ & $\mathrm{IFI}$ & $\mathrm{TLI}$ & $\mathrm{RMSEA}$ & $\mathrm{Cl}(90 \%)$ & $\mathrm{ECVI}$ & $\mathrm{Cl}(90 \%)$ \\
\hline THI-U & & & & & & & & & & \\
1 (25 items) & 1033.97 & 272 & 3.80 & 0.58 & 0.59 & 0.54 & 0.16 & $0.15-0.17$ & 10.46 & $9.6-11.40$ \\
2 (25 items) & 689.02 & 234 & 2.94 & 0.75 & 0.76 & 0.68 & 0.13 & $0.12-0.15$ & 7.99 & $7.3-8.7$ \\
3 (25 items) & 285.84 & 190 & 1.50 & 0.95 & 0.95 & 0.92 & 0.07 & $0.05-0.08$ & 5.1 & $4.71-5.55$ \\
\hline
\end{tabular}

THI-U: Urdu version of Tinnitus Handicap Inventory, CFI: conformity fit index, IFI: incremental fit index, TLI: Tucker-Lewis Index, RMSEA: root-mean- square error of approximation, $\mathrm{Cl}$ : confidence interval, ECVI: expected cross-validation index

removing item 2 statistically significant improved the model fit $\left[\chi^{2}=285.84 ; \chi^{2} / \mathrm{df}=1.50\right.$; conformity fit index $(\mathrm{CFI})=0.95$; incremental fit index $(\mathrm{IFI})=0.95 ; \mathrm{TLI}=0.92$; root-mean-square error of approximation $(\mathrm{RMSEA})=0.07,90 \%$ confidence interval $(\mathrm{CI})=0.05-0.08$; expected cross-validation index $(\mathrm{ECVI})=5.1,90 \% \mathrm{CI}=4.71-5.55]$ due to same response as noted in highly negative correlated error variance associated with those items. According to previous studies [22-24], however, theoretical removals should also be evaluated first through exploratory factor analysis and content analysis.

Table 6 and Fig. 1 reviewed the factor loadings for each item. Only Item No. 2 had a negative estimate. Factor loadings signified the strength of the relationship between the construct and the item in terms of shared variance. Standardized values closer to 1.0 were optimal. In case of cross-loadings (item-2), a lower estimate was expected. These estimates ranged from 0.203 to 0.819 , which were significant and explained the overall goodness of fit of the model.

\section{Discussion}

The current study established that the three-factor structure fits significantly for a final assessment of results. The results of the current analyses revealed that not only the total score but the scores of the three subscales can be used for data analysis, even if there are significant inter-correlations between the subscales. In our study, only item-2 appeared to be problematic and could not correspond with the overall scale.

The current study was also designed to examine the reliability and validity of Urdu translation of the THI in Pakistan and also to validate the factorial structure of the THI with a confirmatory factor analysis. Surprisingly, the current study results hold opposing views with previous studies $[7,9,12,13$, $25,26]$. On the other hand, numerous previous studies findings supported current research results that three factor solution including functional, emotional, and catastrophic subscales were confirmed through confirmatory factor analysis in Pakistani context $[6,27]$. For final assessment of this result it should be considered that cofirmatory factor analysis bears one essential advantage: it provides clear estimates of error variance parameters and it is therefore feasible to assess and
Table 6. Factor loading of confirmatory factor analysis

\begin{tabular}{|c|c|c|c|c|}
\hline \multirow{2}{*}{ Scales } & \multirow{2}{*}{ Items } & \multicolumn{3}{|c|}{ Factors } \\
\hline & & B & SE & $\beta$ \\
\hline \multirow[t]{12}{*}{ Functional } & 1 & 1.000 & 0.000 & $0.565^{* * *}$ \\
\hline & 2 & -0.382 & 0.225 & -0.164 \\
\hline & 4 & 1.193 & 0.228 & $0.620^{* * *}$ \\
\hline & 7 & 1.490 & 0.273 & $0.679^{* * *}$ \\
\hline & 9 & 1.613 & 0.268 & $0.780^{* * *}$ \\
\hline & 12 & 1.366 & 0.215 & $0.705^{* * *}$ \\
\hline & 14 & 1.370 & 0.255 & $0.654^{* * *}$ \\
\hline & 15 & 1.607 & 0.287 & $0.695^{* * *}$ \\
\hline & 18 & 1.604 & 0.314 & $0.695^{* * *}$ \\
\hline & 20 & 1.528 & 0.279 & $0.672^{* * *}$ \\
\hline & 24 & 0.959 & 0.215 & $0.448^{* * *}$ \\
\hline & 13 & 0.980 & 0.241 & $0.474^{* * *}$ \\
\hline \multirow[t]{8}{*}{ Emotional } & 3 & 1.000 & 0.000 & $0.577^{* * *}$ \\
\hline & 6 & 0.980 & 0.206 & $0.524^{* * *}$ \\
\hline & 10 & 1.141 & 0.156 & $0.663^{* * *}$ \\
\hline & 16 & 0.948 & 0.206 & $0.596^{* * *}$ \\
\hline & 17 & 0.375 & 0.185 & $0.203^{*}$ \\
\hline & 22 & 1.166 & 0.189 & $0.748^{* * *}$ \\
\hline & 25 & 1.182 & 0.216 & $0.643^{* * *}$ \\
\hline & 21 & 1.097 & 0.179 & $0.691^{* * *}$ \\
\hline \multirow[t]{5}{*}{ Catastrophic } & 5 & 1.000 & 0.000 & $0.819^{* * *}$ \\
\hline & 8 & 0.772 & 0.114 & $0.603^{* * *}$ \\
\hline & 11 & 0.825 & 0.096 & $0.722^{* * *}$ \\
\hline & 19 & 0.561 & 0.109 & $0.471^{* * *}$ \\
\hline & 23 & 0.667 & 0.100 & $0.595^{* * *}$ \\
\hline
\end{tabular}

$* p<0.001, * * * p<0.0001$. SE: standard error

correct the measurement errors. Therefore the results of current analyses allow the conclusion that not only the total score but also the scores of the three subscales can be considered for data analysis, even if there are considerable inter-correlations between the subscales.

In addition, current study investigated the reliability of the THI-U showing high internal consistency for the overall scale. For the three subscale of THI-U such as functional, emotional and catastrophic moderate reliability was demonstrated in current study. Previous studies on THI have demonstrated a good internal consistency for total of the tinnitus scale along with three domains with Cronbach's alpha between 0.80 to 0.95 . 


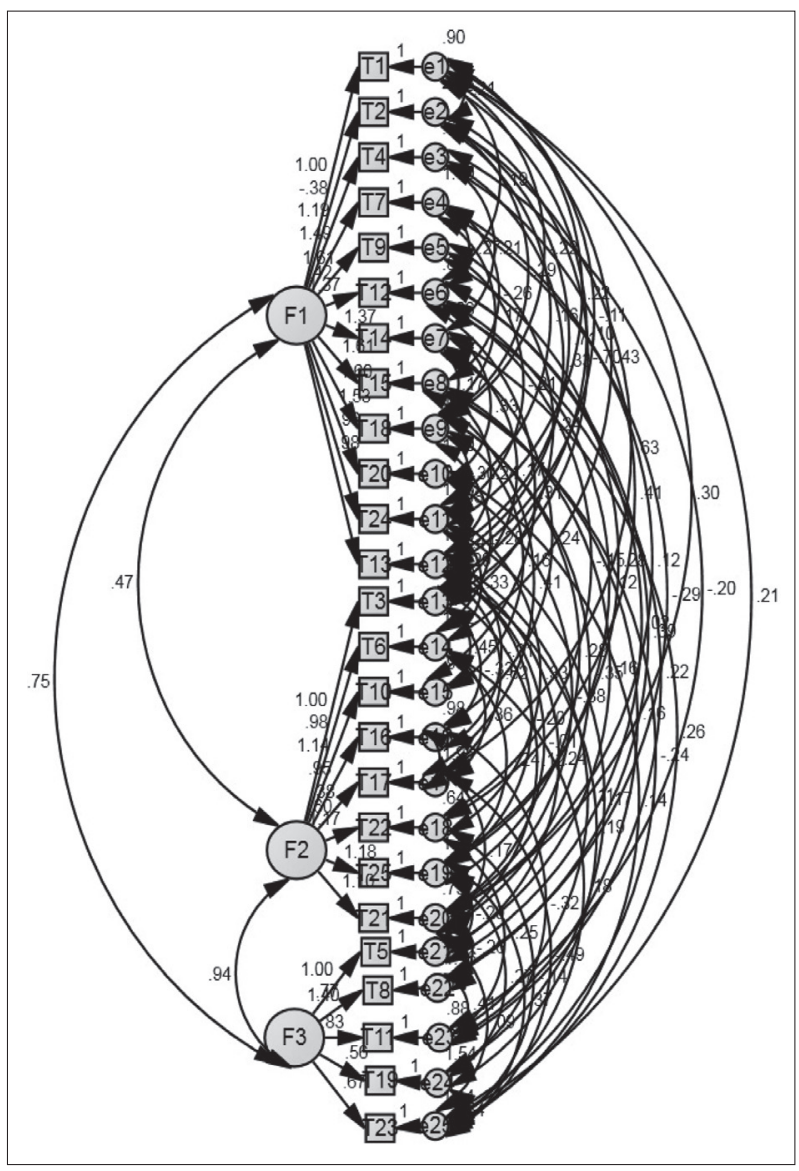

Fig. 1. Path diagram for the three factor varimax model with item loading from the confirmatory factor analysis in main study. F1: functional, F2: emotional, F3: catastrophic.

Danish [7], Spanish [8], German [9], Brazilian Portuguese [10], Turkish [11], Italian [12], Chinese (Cantonese) [13], French [14], Kannada [15], Persian [16], and Hebrew [17]. Similar to the previous studies findings Cronbach's alpha of the total THI-U ( $\alpha=0.93)$, functional $(\alpha=0.86)$, emotional $(\alpha=0.80)$ and catastrophic subscale $(\alpha=0.81)$.

Further this current study scrutinized the construct validity of THI-U. The total of the tinnitus score was positively significant correlated with its own three domains.

\section{Limitations \& recommendations}

Although the current study provides important new knowledge about the validation of the THI-U it also possesses certain limitations as well. First, the pilot was based on a sample of university students for the translation purpose, this was done in order to validate translation and it was necessary to involve a sample that had comprehensive command over English and Urdu language. However, another problem in our study was that it involved only self report measures to validate THI-U. In contrast to results of previous exploratory analyses, the present findings speak for a three-factor in contrast to a unifactorial structure. Results suggested that it is a reliable and valid tool which can be used in all clinical setting to evaluate and quantify the impact of tinnitus on the quality of life of a Pakistani patient. Future research is needed to replicate this result in different tinnitus populations.

Finally, it was obvious that item-2 of the functional subscale of the THI-U does not demonstrate satisfying reliability. This finding was confirmed by previous validation studies in other languages [27]. The reason demonstrated is due to the considerable overlap of item No. 2 with other items like item No. 8, 9 , and 19. Future research should consider either eliminating or adding/modifying this item. This might help in increasing the reliability.

\section{Conclusion}

The statistical findings revealed that THI is a valid and reliable instrument to evaluate tinnitus distress. Many previous studies originally suggested three-factor structure could be confirmed [27]. Although different authors of previous studies believed in a strong overlap between the subscales, which did not justify assessing the scales in parts, the current analyses contradicts many previous findings and conforms findings of [27]. In this study, confirmatory factor analysis allowed us in specifying item loads on factors and excluding cross loadings, the results demonstrated satisfactory incremental validity of subscales. Hence it is suggested to use not only the total score but also the subscales for evaluating tinnitus distress. Therefore, THI-U can present important information about precise facets of tinnitus distress along with diagnostic interviews in clinical practice. Because of the international importance of the THI, there should be future research that tries to replicate this three-factor structure of the THI in other languages as well as in different tinnitus populations to further validate our findings.

\section{Conflicts of interest}

The authors have no financial conflicts of interest.

\section{REFERENCES}

1) Habets B. The complete guide to tinnitus: a practical guide to understanding and treating tinnitus. London: Carnell;1995.

2) Shargorodsky J, Curhan GC, Farwell WR. Prevalence and characteristics of tinnitus among US adults. Am J Med 2010;123:711-8.

3) Han BI, Lee HW, Kim TY, Lim JS, Shin KS. Tinnitus: characteristics, causes, mechanisms, and treatments. J Clin Neurol 2009;5:11-9.

4) Lee SY, Kim JH, Hong SH, Lee DS. Roles of cognitive characteristics in tinnitus patients. J Korean Med Sci 2004;19:864-9.

5) Crummer RW, GA Hassan. Diagnostic approach to tinnitus. Am Fam Physician 2004;69:120-6.

6) Newman CW, Jacobson GP, Spitzer JB. Development of the Tinnitus Handicap Inventory. Arch Otolaryngol Head Neck Surg 1996;122: 
143-8.

7) Zachariae R, Mirz F, Johansen LV, Andersen SE, Bjerring P, Pedersen CB. Reliability and validity of a Danish adaptation of the Tinnitus Handicap Inventory. Scand Audiol 2000;29:37-43.

8) Herráiz C, Plaza G, Tapia M. Handicap evaluation in tinnitus patients. Acta Otorrinolaringol Esp 2001;52:534-8.

9) Kleinjung T, Fischer B, Langguth B, Sand PG, Hajak G, Dvorakova J, et al. Validation of the German-version Tinnitus Handicap Inventory. Psychiatr Prax 2007;34(S 1):S140-2.

10) Schmidt LP, Teixeira VN, Dall'Igna C, Dallagnol D, Smith MM. Brazilian Portuguese language version of the "Tinnitus Handicap Inventory”: validity and reproducibility. Braz J Otorhinolaryngol 2006;72: 808-10.

11) Aksoy S, Firat $Y$, Alpar R. The Tinnitus Handicap Inventory: a study of validity and reliability. Int Tinnitus J 2007;13:94-8.

12) Monzani D, Genovese E, Marrara A, Gherpelli C, Pingani L, Forghieri M, et al. Validity of the Italian adaptation of the Tinnitus Handicap Inventory; focus on quality of life and psychological distress in tinnitus-sufferers. Acta Otorhinolaryngol Ital 2008;28:126-34.

13) Kam AC, Cheung AP, Chan PY, Leung EK, Wong TK, van Hasselt $\mathrm{CA}$, et al. Psychometric properties of the Chinese (Cantonese) Tinnitus Handicap Inventory. Clin Otolaryngol 2009;34:309-15.

14) Ghulyan-Bédikian V, Paolino M, Giorgetti-D'Esclercs F, Paolino F. [Psychometric properties of a French adaptation of the Tinnitus Handicap Inventory]. Encephale 2010;36:390-6.

15) Zacharia T, Naik PV, Sada S, Kuniyil JG, Dwarakanath VM. Development and standardization of Tinnitus Handicap Inventory in Kannada. Int Tinnitus J 2012;17:117-23.

16) Jalali MM, Soleimani R, Fallahi M, Aghajanpour M, Elahi M. Psy- chometric properties of the Persian version of the Tinnitus Handicap Inventory (THI-P). Iran J Otorhinolaryngol 2015;27:83-94.

17) Oron $Y$, Shushan S, Kreitler S, Roth Y. A Hebrew adaptation of the Tinnitus Handicap Inventory. Int J Audiol 2011;50:426-30.

18) Sardar S, Wahab A. Optical character recognition system for Urdu. Proceedings of the Information and Emerging Technologies (ICIET), 2010 International Conference; 2010 Jun 14-16: Karachi. New York, NY: IEEE;2010.

19) Brislin RW. Translation: applications and research. New York, NY: Gardner Press; 1976.

20) Hambleton RK. Guidelines for adapting educational and psychological tests: a progress report. Eur J Psychol Assess 1994;10:229-44.

21) Newman CW, Sandridge SA, Jacobson GP. Psychometric adequacy of the Tinnitus Handicap Inventory (THI) for evaluating treatment outcome. J Am Acad Audiol 1998;9:153-60.

22) Osborne JW. Best practices in quantitative methods. Thousand Oaks, CA: SAGE Publications, Inc.;2008.

23) Tabachnick BG, Fidell LS. Using multivariate statistics. Boston, MA: Allyn and Bacon;2001.

24) Urbina S. Essentials of psychological testing. 2nd ed. Hoboken, NJ: John C Wiley \& Sons, Inc.;2004.

25) Meng Z, Zheng Y, Liu S, Wang K, Kong X, Tao Y, et al. Reliability and validity of the chinese (mandarin) Tinnitus Handicap Inventory. Clin Exp Otorhinolaryngol 2012;5:10-6.

26) Baguley DM, Andersson G. Factor analysis of the Tinnitus Handicap Inventory. Am J Audiol 2003;12:31-4.

27) Kleinstäuber M, Frank I, Weise C. A confirmatory factor analytic validation of the Tinnitus Handicap Inventory. J Psychosom Res 2015; 78:277-84. 